\title{
Feasibility of a septal mucosal flap for preventing re-stenosis following the Draf III procedure
}

\author{
O ERDUR, K OZTURK, K ERKAN
}

Department of Otolaryngology, Selcuk University, Konya, Turkey

\begin{abstract}
Background: Re-stenosis and a consequent need for revision surgery are the most common problems in the follow-up period following endoscopic modification of the Lothrop procedure.

Method: This paper reports a new technique for reconstructing and resurfacing of the posterior frontal recess bone for prevention of re-stenosis.

Results: A 46-year-old man presented with a frontal sinus osteoma, and treatment featured an endoscopic modification of the Lothrop procedure. A vascularised, posteriorly based, septal mucosal flap was used in reconstruction. There have been no reported issues over 24 months of follow up.

Conclusion: The use of a nasoseptal flap seems feasible to reduce scarring and recurrence of (common) frontal recess stenosis after a Draf III operation.
\end{abstract}

Key words: Endoscopy; Frontal Sinus; Nasal Surgical Procedures; Surgical Flaps; Paranasal Sinus Diseases; Postoperative Complications

\section{Introduction}

In 1991, Draf described a transnasal endoscopic/microscopic modification of the endoscopic modified Lothrop procedure that was termed the Draf III technique. ${ }^{1}$ In this procedure, a large view is created for frontal sinus pathologies, and nasofrontal communication is maximised by resecting the floor of the sinus, the inter-sinus septum and the upper nasal septum. Chin et al. described an outside-in approach form of the endoscopic modified Lothrop procedure, which allows easier access to the sinus and reduces the operation time. ${ }^{2}$

Although the efficacy of the endoscopic modified Lothrop procedure is well established, re-stenosis and a consequent need for revision are the most common problems in the follow-up period. Stenosis of the neo-ostium of the frontal sinus is caused by adhesions, scarring, polyp formation or neo-osteogenesis. ${ }^{3,4}$ The incidence of neo-ostial stenosis varies widely in published reports.

Some new techniques and applications seek to prevent restenosis, but no consensus has emerged on how this can best be done. A nasoseptal flap, based on the nasoseptal artery, was described by Hadad et al. in 2006. ${ }^{5}$ This flap has found wide applications in various types of skull base surgery.

Here, we describe, for the first time, the use of this nasoseptal flap technique to reconstruct the frontal neo-ostium after an endoscopic modified Lothrop procedure and summarise the recent literature.

\section{Case report}

A 46-year-old man was admitted to our clinic because of pain in the head and left eye. His eye movements and vision were normal, but exophthalmos was evident. The problems had developed over the prior one year. At that time, the patient had been operated on to treat a lesion in the same area. Both endoscopic surgery and trephination had been employed, and the histopathological diagnosis was an osteoma. No other relevant pathology was present. The other results of the physical examination were normal.

Computed tomography of the paranasal sinus revealed a hyperdense mass approximately $2 \times 2 \mathrm{~cm}$ in size, extending to the left orbit from the frontoethmoidal recess. This mass pushed the medial rectus muscle laterally, and seemed to be a recurrent osteoma (Figure 1). The left frontal sinus was obliterated with soft tissue because the natural ostium was completely filled with the osteoma.

The operation (performed under general anaesthesia) featured the outside-in endoscopic modified Lothrop procedure as described by Chin et $a l^{2}$ Resection of the frontal sinus floor proceeded from the lamina papyracea to the contralateral lamina papyracea and the superior nasal septum. The inter-orbital distance was created to $2.5 \mathrm{~cm}$. Such a wide view allowed total excision of the osteoma from the orbit and the frontal sinus, without any complications.

A nasoseptal flap was prepared from the right nasal cavity prior to the Draf procedure. The neurovascular pedicled flap of the nasal septum was based on the nasoseptal artery, a branch of the posterior septal artery described by Hadad et al. (Figure 2). ${ }^{5}$ In order to achieve the longest size of flap, the vertical incision of the flap was carried out at the distal edge of septal cartilage. Thereafter, the mucoperichondrium and the mucoperiosteal flap were elevated and preserved posteriorly in the nasopharyngeal area (Figure 3). 


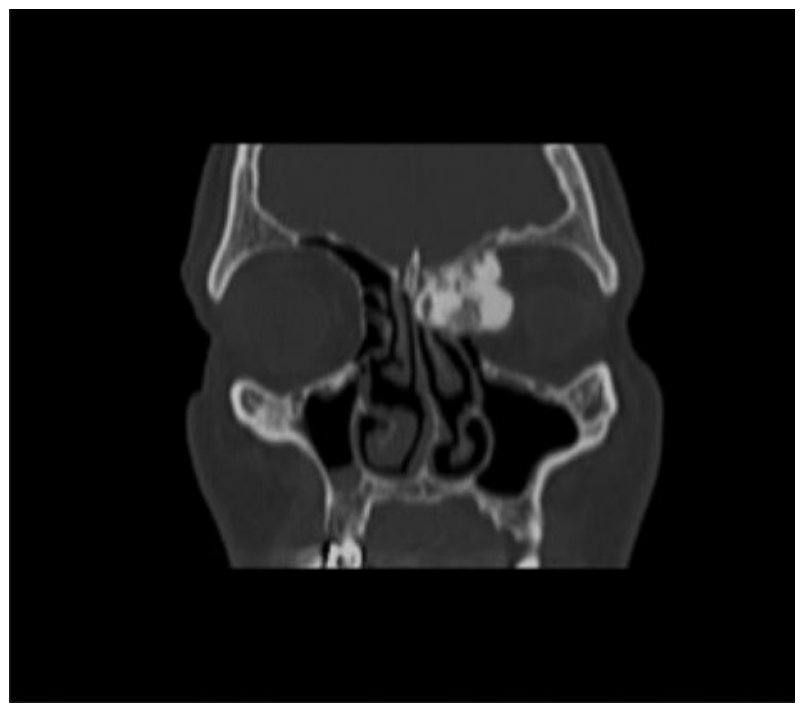

FIG. 1

Pre-operative coronal computed tomography image of the paranasal sinus.

After the Draf procedure and removal of the tumour, the flap was used to cover the posterior wall of the sinus and the common recess (Figure 4). The flap was packed with gel foam and supported by a 12-French Foley catheter balloon. Nasal silicon buffers were inserted on both sides of the septum.

On the 2nd post-operative day, the Foley catheter was removed and the patient was discharged with a prescription for amoxicillin/clavulanic acid. Rinsing of the nasal cavity commenced on the 5th post-operative day, after removal of the nasal silicon buffers.

No major complication was reported during or after the operation. At the 24-month follow up, no recurrence of the osteoma was evident and the common recess was no smaller than 50 per cent of that at the end of surgery (Figures 5 and 6).

\section{Discussion}

The Draf III technique creates a median, common, frontal drainage pathway. Many experts have verified that this

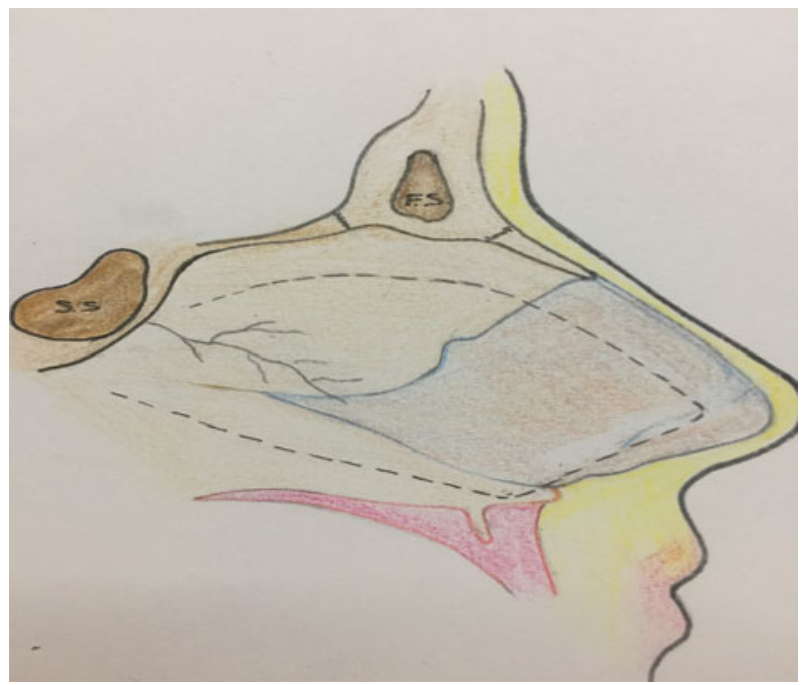

FIG. 2

Drawing of the nasoseptal flap which is planned on the septum.

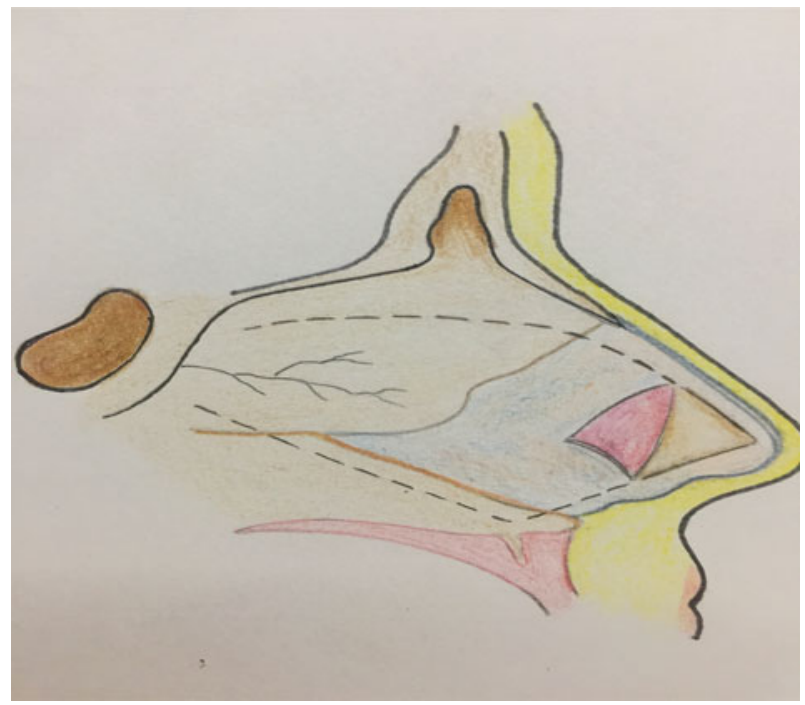

FIG. 3

Drawing of the distal vertical incision and elevation to achieve the longest flap size.

procedure is an efficient alternative to external approaches. ${ }^{2,3}$ Stenosis of the frontal neo-ostium after a Draf III procedure remains the most common complication and the principal trigger of a need for recurrent surgery. Although the frequency varies among different studies in the literature, neo-ostial re-stenosis is not uncommon.

Shirazi et al. reported the outcomes of 97 patients who underwent Draf III surgery; 23 per cent required revision surgery to treat re-stenosis of the ostium within a mean follow-up time of 1.5 years. $^{6}$ Anderson and Sindwani reviewed the literature on the endoscopic modified Lothrop procedure (18 studies with 612 patients); 19 per cent of patients developed re-stenosis of the nasofrontal ostium, and revision surgery was needed by 14 per cent of these patients. $^{7}$

Various approaches and strategies have been developed in efforts to prevent this complication, including extension of the neo-ostium, the use of various pharmacological agents, stents for the neo-ostium, new surgical techniques and the use of free mucosal grafts. However, re-stenosis remains challenging and no consensus on prevention has emerged.

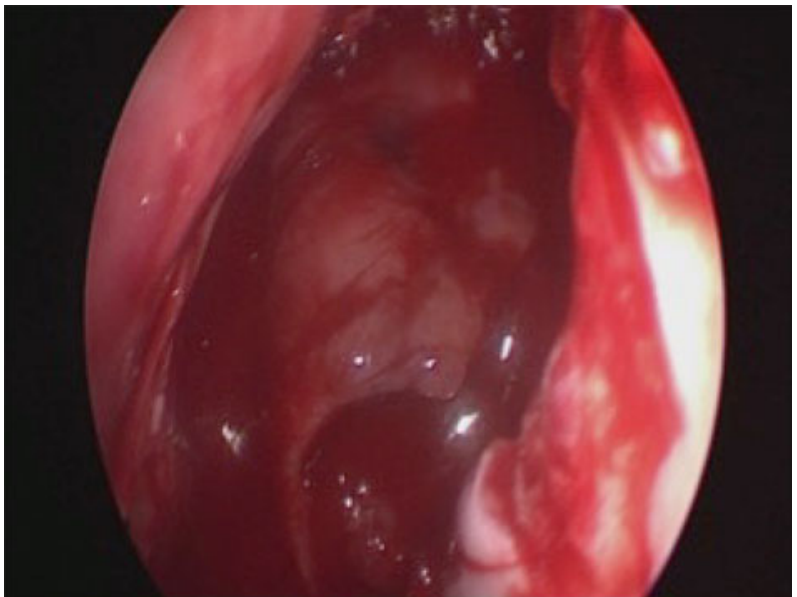

FIG. 4

Endoscopic image of the patient during surgery. 


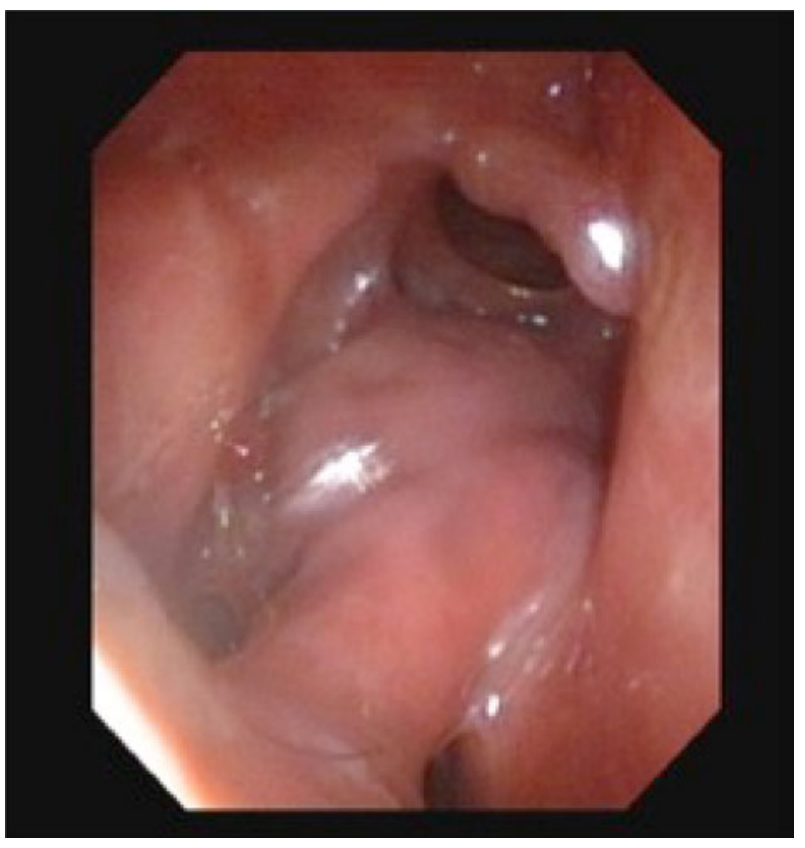

FIG. 5

Endoscopic image of the patient 24-months post-operatively.

Recently, Seyedhadi et al. published a preliminary report on the use of a septal flap based on the anterior ethmoidal artery after a Draf III procedure. ${ }^{8}$ Flap use was strongly recommended to reduce re-stenosis of the recess.

Nasoseptal flaps based on the posterior nasoseptal artery, as defined by Hadad et al., have been used with various aims in endoscopic surgery. ${ }^{5}$ To the best of our knowledge, such flaps have never been placed after an endoscopic modified Lothrop procedure for preventing re-stenosis. Jones et al. used this flap for resurfacing frontal defects, such as after cerebrospinal fluid leak, in 2012. ${ }^{9}$ In a recent cadaver study, AlQahtani et al. found that a posteriorly based nasoseptal flap could be used after the endoscopic modified Lothrop procedure. ${ }^{10}$ The authors also described a laterally based nasoseptal flap for use during the endoscopic modified

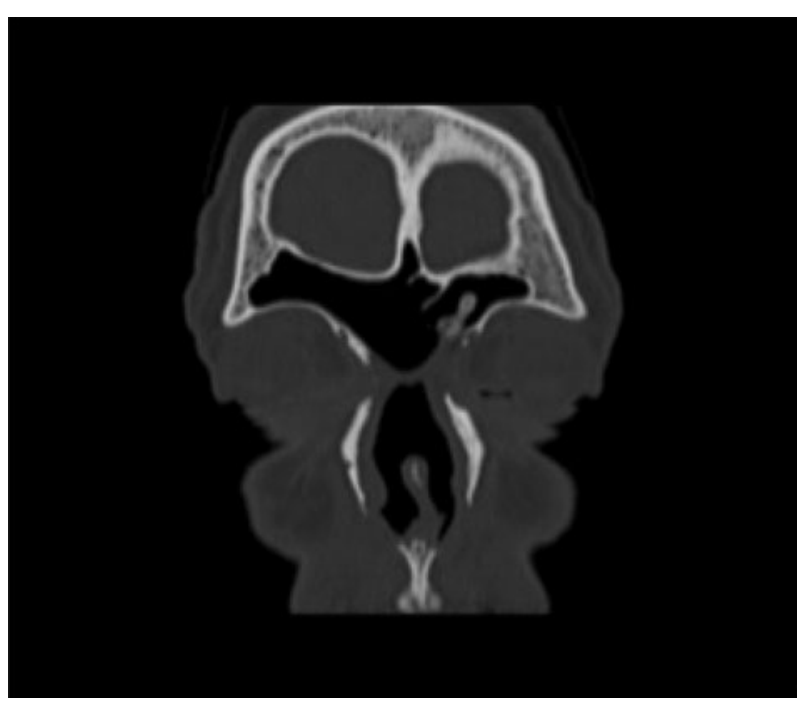

FIG. 6

Coronal computed tomography image of the paranasal sinus at 24-months post-operatively.
Lothrop procedure, and suggested that both posteriorly and laterally based flaps should be employed to cover both the anterior and posterior walls of the frontal sinus.

The use of a posterior nasoseptal flap affords certain advantages during an endoscopic modified Lothrop procedure. Elevation of the right septal perichondrium and periosteum (to place the flap) affords a blood-free endoscopic view. After preparation, the flap is placed to run down to the nasopharynx. Thus, the flap does not block the image, unlike laterally based flaps. The pedicle of the flap extends over and covers part of the skull base, preventing latent cerebrospinal fluid leakage.

- The Draf III procedure creates a large view for frontal sinus pathologies

- In addition, nasofrontal communication is maximised by resecting the sinus floor, inter-sinus septum and upper nasal septum

- Re-stenosis and a need for revision surgery are the most common problems after the procedure

- This paper presents a technique for reconstructing and resurfacing posterior frontal recess bone to prevent re-stenosis

- A frontal sinus osteoma patient was treated with Draf III; a vascularised, posteriorly based, septal mucosal flap was used in reconstruction

- This nasoseptal flap prevented re-stenosis after Draf III, and may reduce scarring and common recess stenosis

Studies on endoscopic modified Lothrop procedure outcomes use inconsistent definitions of neo-ostial stenosis. In most studies, recess re-stenosis is defined as a reduction of over 50 per cent in the sectional surface area of the recess compared to that at the end of surgery. ${ }^{3,8}$ We evaluated neo-ostial patency using a curved suction tip and found that recess stenosis was less than 50 per cent. Thus, the technique was successful; no re-stenosis was detected during 24-months' follow up. In this case, after an outside-in endoscopic modified Lothrop procedure, the frontal sinus ostium was reconstructed using a nasoseptal flap that prevented re-stenosis of the neo-ostium.

\section{Conclusion}

We present the first report of a nasoseptal flap used to prevent re-stenosis after an endoscopic modified Lothrop procedure. Our success may pave the way for flap use during other endoscopic modified Lothrop procedures, as this appears to be a safe and easy way to manage re-stenosis. Additional research on more patients is necessary to validate our findings.

\section{References}

1 Draf W. Endonasal micro-endoscopic frontal sinus surgery: the Fulda concept. Oper Tech Otolaryngol Head Neck Surg 1991;2: 234-40

2 Chin D, Snidvongs K, Kalish L, Sacks R, Harvey RJ. The outside-in approach to the modified endoscopic Lothrop procedure. Laryngoscope 2012;122:1661-9

3 Wormald PJ. Salvage frontal sinus surgery: the endoscopic modified Lothrop procedure. Laryngoscope 2003;113:276-83

4 Rajapaksa SP, Ananda A, Cain TM, Oates L, Wormald PJ. Frontal ostium neo-osteogenesis and restenosis after modified 
endoscopic Lothrop procedure in an animal model. Clin Otolaryngol Allied Sci 2004;29:386-8

5 Hadad G, Bassagasteguy L, Carrau RL, Mataza JC, Kassam A, Snyderman $\mathrm{CH}$ et al. A novel reconstructive technique after endoscopic expanded endonasal approaches: vascular pedicle nasoseptal flap. Laryngoscope 2006;116:1882-6

6 Shirazi MA, Silver AL, Stankiewicz JA. Surgical outcomes following the endoscopic modified Lothrop procedure. Laryngoscope 2007; 17:765-9

7 Anderson P, Sindwani R. Safety and efficacy of the endoscopic modified Lothrop procedure: a systematic review and meta-analysis. Laryngoscope 2009;119:1828-33

8 Seyedhadi S, Mojtaba MA, Shahin B, Hoseinali K. The Draf III septal flap technique: a preliminary report. Am J Otolaryngol 2013;34:399-402

9 Jones V, Virgin F, Riley K, Woodworth BA. Changing paradigms in frontal sinus cerebrospinal fluid leak repair. Int Forum Allergy Rhinol 2012;2:227-32

10 AlQahtani A, Bignami M, Terranova P, Digilio E, Basilico F, Abdulrahman $\mathrm{S}$ et al. Newly designed double-vascularized nasoseptal flap to prevent restenosis after endoscopic modified Lothrop procedure (Draf III): laboratory investigation. Eur Arch Otorhinolaryngol 2014;271:2951-5

Address for correspondence:

Dr Omer Erdur

Department of Otolaryngology,

Selcuk University Medical School,

Alaeddin Keykubad Campus,

42075 Yeni Istanbul Caddesi,

Konya, Turkey

E-mail: dromerdur@yahoo.com

Dr O Erdur takes responsibility for the integrity of the content of the paper

Competing interests: None declared 